\title{
Day and night pain measurement in rheumatoid arthritis
}

\author{
B Rojkovich, T Gibson
}

\begin{abstract}
Objective-An attempt was made to see if rheumatoid arthritis (RA) patients can use visual analogue scales (VAS) to distinguish and grade the severity of pain at night, during rest, and on joint movement and to determine if discriminate measurement of these three pain components enhances the value of VAS estimation.

Methods-Two hundred and fifty two consecutive RA patients were evaluated by a single observer using $10 \mathrm{~cm}$ VAS for pain at night, at rest during the day, and on movement. Values were correlated against age, disease duration, joint tenderness, swollen joint count, erythrocyte sedimentation rate (ESR), $C$ reactive protein (CRP), and Larsen $x$ ray scores.

Results-Night pain was recorded by 71 $(28 \%)$ and this component of pain was lower than VAS scores for daytime rest and movement. However, those with nocturnal pain had significantly more joint tenderness $(\mathbf{p}<0.0001)$, swollen joints $(\mathbf{p}<0.0001)$, and higher ESR and CRP. Age, disease duration, and radiographic scores were similar in those with and without night pain. Correlations of joint tenderness were apparent for all three pain scores but only nocturnal pain correlated with swollen joints $(p<0.001)$ and CRP $(p<0.005)$. Age, disease duration, and radiographic severity correlated with daytime rest or movement scores but not nocturnal pain.

Conclusion-Patients were able to distinguish and estimate the severity of pain at rest, on movement, and at night. The occurrence of night pain characterised those with more active disease and night pain VAS measurement correlated best with measures of joint inflammation whereas daytime pain scores, both at rest and on movement, seemed influenced by the degree of permanent joint damage. Thus, discrete measurement of rest, movement, and nocturnal pain may provide useful information about RA disease status.

(Ann Rheum Dis 1998;57:434-436)
\end{abstract}

For those with rheumatoid arthritis (RA), pain is the most important symptom. ${ }^{1}$ It is the strongest determinant of disability ${ }^{2-3}$ and appropriately it is the feature most often used to follow the activity and course of the disease. ${ }^{4}$ Measurement of pain is achieved by various means including self evaluation of descriptive and figurative scales and actual joint tenderness. . $^{5-7}$

One of the most popular methods of self evaluation is the $10 \mathrm{~cm}$ visual analogue scale (VAS). This has a long pedigree and although its precision, sensitivity, and reproducibility have been questioned, ${ }^{8-10}$ its ease of use has ensured its common application to clinical practice and research. It requires patients to indicate on a line the maximum level of pain intensity experienced during a prescribed period. No distinction is usually made between discomfort at rest and activity nor of diurnal fluctuations of pain. There is evidence that patients can identify different pain sensations at rest and on activity and that RA activity follows a circadian rhythm with worsening of the illness during the night. ${ }^{11}{ }^{12}$ We therefore attempted to examine whether a VAS pain scale could discriminate between pain during the day and night and between rest and activity pain. We also determined to what extent each was related to other indices of inflammatory disease activity and severity.

\section{Methods}

Two hundred and fifty two outpatients with RA, according to the 1987 ARA revised criteria as defined by the American College of Rheumatology ${ }^{13}$ were examined by a single clinician. Patients were seen consecutively and the nature of the study explained. They were asked to indicate their pain severity on three 10 $\mathrm{cm}$ horizontal VAS each labelled "no pain" at one end and "worst possible pain" at the other. Patients were asked to note their worst daytime pain at rest and during joint movement over the preceding seven days and also any nocturnal pain experienced. If patients were not awoken at night they were advised to mark the nocturnal VAS at the "no pain" point.

In addition, joint tenderness was determined by the Ritchie Articular Index. ${ }^{6}$ The number of joints with soft tissue swelling was counted, a total of 44 joints being included. Westergren erythrocyte sedimentation rate (ESR) and C reactive protein (CRP) were measured in the hospital laboratory. Active disease was defined
Accepted for publication 12 May 1998 
Table 1 Clinical features and characteristics of patients with and without nocturnal joint pain. Data shown as median values with ranges in parentheses

\begin{tabular}{llll}
\hline & Night pain & No night pain & p Value \\
\hline Male & 24 & 10 & ) \\
Female & 157 & 61 & ) $0.97\left(\chi^{2}=0.0\right)$ \\
Total & $181(72 \%)$ & $71(28 \%)$ & \\
Age & $57(19-80)$ & $54(24-74)$ & 0.56 \\
Disease duration (y) & $8(1-37)$ & $7(1-46)$ & 0.36 \\
Joint tenderness & $16(3-35)$ & $8(0-29)$ & 0.0001 \\
Swollen joints (n) & $6(0-14)$ & $4(1-10)$ & 0.0001 \\
ESR & $35(1-115)$ & $30(1-100)$ & 0.052 \\
CRP & $7(1-80)$ & $4(1-66)$ & 0.028 \\
Radiographic score & $43(0-132)$ & $40(10-123)$ & 0.19 \\
\hline
\end{tabular}

Table 2 Spearman correlation of three pain scores with other clinical variables

\begin{tabular}{lllll}
\hline & & $\begin{array}{l}\text { Daytime } \\
\text { movement }\end{array}$ & Daytime rest & Nocturnal \\
\hline Joint tenderness & $r$ & 0.246 & 0.250 & \\
Swollen joints (n) & $\mathrm{p}$ & $<0.0001$ & $<0.0001$ & $<.357$ \\
& $r$ & 0.025 & 0.027 & 0.00001 \\
CRP & $\mathrm{p}$ & $\mathrm{NS}$ & $\mathrm{NS}$ & $<0.001$ \\
& $\mathrm{r}$ & 0.064 & 0.078 & 0.202 \\
ESR & $\mathrm{p}$ & $\mathrm{NS}$ & $\mathrm{NS}$ & $<0.005$ \\
& $\mathrm{r}$ & 0.169 & 0.180 & 0.252 \\
Radiographic score & $\mathrm{p}$ & $<0.01$ & $<0.01$ & $<0.0001$ \\
& $\mathrm{r}$ & 0.089 & 0.155 & 0.078 \\
Age & $\mathrm{p}$ & $\mathrm{NS}$ & $<0.05$ & $\mathrm{NS}$ \\
& $\mathrm{r}$ & 0.136 & 0.190 & 0.015 \\
Disease duration & $\mathrm{p}$ & $<0.05$ & $<0.005$ & $\mathrm{NS}$ \\
& $\mathrm{r}$ & 0.159 & 0.021 & -0.127 \\
& $\mathrm{p}$ & $<0.05$ & $\mathrm{NS}$ & $<0.05$ \\
\hline
\end{tabular}

Table 3 The odds ratio (95\% confidence intervals) of those with high disease activity among patients with and without nocturnal pain. (High disease activity defined as six or more tender joints, three or more swollen joints, ESR $>28 \mathrm{~mm} 1 \mathrm{st} h, C R P>20 \mathrm{mg} / \mathrm{l})$. The odds ratio for radiographic scores above the median value are also included in the table

\begin{tabular}{lll}
\hline & $\begin{array}{l}\text { Odds } \\
\text { ratio }\end{array}$ & $\begin{array}{l}95 \% \\
\text { Confidence } \\
\text { intervals }\end{array}$ \\
\hline Joint tenderness & 7.88 & $(3.23,19.18)$ \\
Swollen joints (n) & 4.61 & $(2.41,8.79)$ \\
CRP & 2.36 & $(1.19,4.70)$ \\
ESR & 2.15 & $(1.20,3.85)$ \\
Radiographic score & 0.94 & $(0.46,1.94)$ \\
\hline
\end{tabular}

as six or more tender joints, three or more swollen joints, ESR more than $28 \mathrm{~mm} 1 \mathrm{st} \mathrm{h}$, CRP more than $20 \mathrm{mg} / \mathrm{l}$.

Radiographic scores were also determined by the same observer using standard Larsen films of hands and feet. ${ }^{14}$

None of the variables fitted a normal distribution because the population inclined toward either active or inactive disease. Comparisons were made by Mann-Whitney U tests for non-parametric data. Spearman rank correlation coefficient was used to estimate the relations between pain and other indices of RA disease activity, severity, age, and disease duration. All subjects were incorporated in this analysis, those without night pain scoring zero for this variable. The odds ratios $(95 \%$ confidence intervals) for the presence of active disease as defined for each measurement were calculated between those with and without nocturnal pain.

\section{Results}

Thirty four male and 218 female RA patients with a median age of 54 years were examined. Daytime movement pain was experienced by
$251(99 \%)$, daytime rest pain by 232 (92\%), and nocturnal pain by $181(72 \%)$. The median (range) VAS of pain on movement was 56 (0-99), daytime rest pain 43 (0-91), and night pain $34(0-99)$. There was no significant difference between daytime rest and nocturnal pain $(p=0.78)$ but movement pain was significantly more severe than both daytime rest pain $(p<0.001)$ and nocturnal pain $(p<0.001)$. Those with nocturnal pain had significantly worse joint tenderness, more swollen joints, and higher ESR and CRP measurements (table 1). Radiological scores, sex distribution, age and the disease duration were not significantly different.

Correlations were apparent between joint tenderness and all three categories of pain scores (table 2). The number of swollen joints and CRP measurements correlated only with nocturnal pain. Using the Bonferroni correction for multiple comparisons each of these correlations and that for multiple comparisons with ESR remained significant $(\mathrm{p}<0.05)$. The ESR correlated with all three pain scores but Larsen $x$ ray scores correlated with daytime rest pain only. Age correlated with daytime rest and activity scores. Disease duration correlated with daytime activity pain and inversely with nocturnal pain.

The odds ratios of high disease activity markers in patients with nocturnal pain were 2.15-7.88 times higher than in patients without nocturnal pain (table 3 ).

\section{Discussion}

Our results confirm that it is possible for patients to discriminate between pain at night and during rest or movement in the daytime. The most severe pain was related to joint movement and the least was that experienced at night. Nevertheless, the experience of nocturnal pain is a distinguishing characteristic of RA compared with other inflammatory joint disorders. ${ }^{15}$

A substantial minority was disturbed by night pain. The circadian variation of disease activity with more joint tenderness ${ }^{11}$ and stiffness $^{12}$ at night, was not reflected by higher nocturnal pain scores in our study. However, previous investigations of this phenomenon have focused mainly on stiffness and laboratory indices rather than pain severity. It is possible that many of our patients were reporting the experience of stiffness as compared with pain because we did not attempt to distinguish between these sensations and patients do have difficulty in discriminating one from the other. Despite the less severe nature of night pain compared with the day, its occurrence was clearly associated with more active disease as reflected by greater joint tenderness, swelling, and CRP values. The severity of radiological damage was not significantly different between these two groups. Thus the presence of pain that awakens patients distinguishes those with the most joint inflammation but not those with the worst radiological joint damage.

The correlation coefficients were often small but nevertheless, the statistically significant positive correlations of day and night pain with 
both joint tenderness and ESR accord with previous observations. ${ }^{3}$ However, the better correlation with nocturnal pain scores and the strong association of night pain with more active disease suggest that discriminatory nocturnal pain measurement by VAS may provide a more refined index of disease activity. This interpretation is supported by the positive correlations of the number of swollen joints and serum CRP measurements with night pain and the absence of similar relations with daytime rest and movement pain. The odds ratios favoured the view that in general, any degree of nocturnal pain was a feature of active RA as measured by joint swelling and the acute phase response. A weak correlation of radiographic scores was confined to daytime rest pain.

The relation of increasing age and disease duration with daytime movement and rest pain and the inverse correlation of disease duration with nocturnal pain reinforce the proposition that night time discomfort is a reflection of disease activity and is less evident in patients with longstanding RA, when the inflammatory features of the disease are more likely to have become quiescent in response to treatment or time.

Previous investigations of pain rating scales have not attempted to discriminate between rest, movement, and nocturnal pain but our study suggests that such distinctions are not only possible but may help to determine the relative contribution of joint inflammation to the total pain experience.
1 Gibson T, Clark B. Use of simple analgesics in rheumatoid arthritis. Ann Rheum Dis 1985;44:27-9.

2 Gordon P, Jones H, Gibson T. The relative importance of pain and $\mathrm{x}$-ray damage to disability in long-standing RA. Br J Rheumatol 1995;34 (suppl 2):12.

3 van Leeuwen $M$, van der Heijde $D$, van Rijswijk $M$, Houtman P, van Riel P, van de Putte L, et al. Interrelationship of outcome measures and process variables in early heumatoid arthritis. A comparison of radiologic damage, physical disability, joint counts and acute phase reactants. J Rheumatol 1994;21:425-29.

4 Stucki G. Towards clinical quality management: rheumatoid arthritis as a case in point. Rheumatology in Europe 1996; 25:97-103.

5 Callahan L, Brooks R, Summey J, Pincus T. Quantitative pain assessment for routine care of rheumatoid arthritic patients using a pain scale based on activities of daily living and a visual analog pain scale. Arthritis Rheum 1987;40: 630-6.

6 Ritchie D, Boyle J, McInnes J, Jasani M, Dalakos T, Grieveson $\mathrm{P}$, et al. Clinical studies with an articular index for the assessment of joint tenderness in patients with rheumatoid arthritis. Q J Med 1968;37:393-406.

7 Charter R, Nehemkis A, Keenan M, Person D, Prete P. The nature of arthritis pain. Br J Rheumatol 1985;24:53-60.

8 Scott J, Huskisson E. Graphic representation of pain. Pain 1976;2:175-84

9 Jensen P, Karoly P, Braver S. The measurement of clinical pain intensity: A comparison of six methods. Pain 1986;27: $117-26$.

10 Downie W, Leatham P, Rhind V, Pickup M, Wright V. The visual analogue scale with assessment of grip strength. Ann Rheum Dis 1978;37:382-4.

11 Harkness J, Richter M, Panayi G, van de Pette K, Unger A, Pownall $\mathrm{R}$, et al. Circadian variation in disease activity in rheumatoid arthritis. BMJ 1982;284:551-3.

12 Bellamy N, Sothern R, Campbell J, Buchanan W. Circadian rhythm in pain, stiffness and manual dexterity in Rheumatoid arthritis: relation between discomfort and disability. Ann Rheum Dis 1991;50:243-8.

13 Arnett F, Edworthy S, Bloch D, McShane D, Fries J, Cooper N, et al. The American Rheumatism Association 1987 revised criteria for the classification of rheumatoid arthritis. Arthritis Rheum 1988;31:315-24.

14 Larsen A, Dale K, Eek M. Radiographic evaluation of rheumatoid arthritis and related conditions by standard matoid arthritis and related conditions by standar
reference films. Acta Radiol Diagnosis 1977;18:481-91.

15 La Montagna G, Tirri R, Baruffo A, Preti B. Viaggi S. Clinical pattern of pain in rheumatoid arthritis. Clin Exp Rheumatol 1997;15:481-5. 